\title{
Virulence in clonal plants: conflicting selection pressures at work?
}

\author{
Tamara van Mölken · Josef F. Stuefer
}

Received: 18 January 2007/ Accepted: 20 August 2007/Published online: 1 September 2007

(C) Springer Science+Business Media B.V. 2007

Keywords Virulence $\cdot$ Clonal plants $\cdot$ Escape $\cdot$ Sexual reproduction · Pathogen transmission $\cdot$ Multiple infections

The evolution of pathogen virulence has received considerable attention during the last decades (Van Baalen and Sabelis 1995; Frank 1996; Day and Proulx 2004). In this note we will discuss two major theories of virulence evolution in the context of clonal plantpathogen interactions and argue that they can lead to contradictory predictions when applied to very long-lived hosts such as clonally propagating plants. We propose that clonal plants and their pathogens may be especially suitable systems for empirically testing hypotheses of virulence evolution.

A generally accepted theory of virulence evolution is based on the trade-off between pathogen transmission and within-host pathogen reproduction (Frank 1996; Lipsitch and Moxon 1997; Day 2003; Andre and Hochberg 2005), the latter being positively correlated with pathogen virulence (see Table 1 for definitions). Pathogens can benefit for a long time from hosts with low intrinsic mortality rates, provided that they exploit them prudently (low virulence), while severe host exploitation (high virulence) shortens the time during which a pathogen can benefit from the host. This effect can be seen as a mortality cost of virulence (Day and Proulx 2004). As a consequence, high host longevity should select for lower levels of virulence. Conversely, increased virulence should be favoured in shortlived hosts (Gandon et al. 2001 and references therein; Day and Proulx 2004), due to the necessity to spread quickly to new hosts and due to the lower mortality costs of virulence (Ewald 1994; Day and Proulx 2004). Depending on the specific host-pathogen interaction a whole range of evolutionary stable strategies can be expected. The optimal strategy

T. van Mölken $(\bowtie) \cdot$ J. F. Stuefer

Department of Experimental Plant Ecology, Radboud University, Toernooiveld 1, Nijmegen 6525 ED,

The Netherlands

e-mail: T.vanMolken@science.ru.nl

J. F. Stuefer

e-mail: J.Stuefer@science.ru.nl 
Table 1 Glossary of used terms

\begin{tabular}{ll}
\hline Virulence: & $\begin{array}{l}\text { The fitness reduction of a host as a result of pathogen infection } \\
\text { (based on Ewald 1994; Brown et al. 2006). }\end{array}$ \\
Pathogen: & An organism which can infect and cause disease in a host (Brown et al 2006). \\
Multiple infection: & $\begin{array}{l}\text { Simultaneous infection of a host by more than one pathogen, belonging to } \\
\text { the same or different pathogen species. }\end{array}$ \\
\hline
\end{tabular}

balances the above-mentioned costs and benefits resulting in the general prediction that pathogens evolve towards intermediate levels of virulence (Gandon et al. 2001 and references therein; Day 2003). This theory, however, is based on single infection events.

Predictions for virulence evolution change when multiple infections (for a definition, see Table 1) are taken into account, since pathogens have to deal with both the host and one or more other pathogens simultaneously (Van Baalen and Sabelis 1995). The general prediction is that in multiply infected hosts pathogens evolve towards higher levels of virulence than in the case of single infections (Nowak and May 1994; May and Nowak 1995; Brown et al. 2002). Prudent host exploitation, facilitating host longevity, becomes less beneficial for a pathogen if other pathogens simultaneously decrease host longevity (Van Baalen and Sabelis 1995). In addition, within-host competition among pathogens is expected to result in increased host exploitation rates and therefore result in increased overall levels of virulence (Van Baalen and Sabelis 1995; Frank 1996). Van Baalen and Sabelis (1995) proposed that pathogen virulence should also increase in anticipation of multiple infections if the probability of multiple infections is high. Clearly, pathogens in multiply infected hosts face a whole array of evolutionary forces that determine the final optimal strategy in each individual case.

Both of these opposing selection pressures on pathogen virulence may act on clonal plants, which can be extremely long-lived (Oinonen 1967; Kemperman and Barnes 1976; Cook 1985; Steinger et al. 1996) and which spread their mortality risks by partial or full independence of ramets (Eriksson 1993). In principle, individuals of clonal plants can persist as long as the rate of vegetative propagation equals or exceeds the rate at which old parts die off (Thomas 2002). This gives systemic pathogens the possibility to establish a virtually unlimited, lifetime infection in clonal species (Stuefer et al. 2004). The extreme longevity of genetic individuals and their multiple representations in populations should select for decreased levels of pathogen virulence for the following two reasons. First, the trade-off between pathogen transmission and reproduction should impose selection pressures for decreased levels of pathogen virulence in clonal plants. Second, clonal propagation and high longevity is likely to buffer spatio-temporal variability in the genetic make-up of populations. In other words, clonal growth enhances the probability of finding a susceptible host in the vicinity of the initially infected host. Host features which assist local pathogen transmission are expected to select for lower levels of virulence (Boots and Mealor 2007). The nature and magnitude of this effect is likely to depend on the spatial positioning of ramets (i.e., clonal growth form), the genetic diversity of the host plant population, and on the spatio-temporal dynamics of pathogen dispersal.

In contrast to selection for decreased levels of virulence, the longevity of clonal plants is likely to increase the chance for multiple infections and should therefore select for increased pathogen virulence. Van Baalen and Sabelis (1995) state that "If the force of infection is high, and hosts remain exposed long enough, multiple infections are bound to occur". Thus, it seems that both high and low pathogen virulence can be selected for in 
clonal plants and in other long-lived organisms. However, current models on virulence evolution do not explain how these conflicting selection pressures are resolved and what levels of pathogen virulence can be expected to result from opposing selection forces.

When considering both above-mentioned theories, one may conclude that pathogens of clonal plants ought to evolve towards higher levels of virulence, as multiple infection events are bound to occur during the lifetime of a clonal plant and the hypothesized tradeoff between pathogen transmission and pathogen replication is based on single infection events only. Exposure to increasingly virulent pathogens should strongly select for escape strategies of the host plant. Since most clonal plants can reproduce both vegetatively and sexually, a switch to flowering and fruit set may represent a way for clonal plants to evade pathogens. It has been proposed that sexual reproduction should be favoured over asexual propagation in infected plants (Hamilton et al. 1990). Therefore we expect infected clonal plants to show increased sexual reproduction through more rapid and intensive flower production, a faster developmental switch to flowering and increased flower or seed production.

Empirical data to confirm or reject these hypotheses are not available to date. To our knowledge there is no evidence for highly virulent pathogens in clonal plants, neither for increased flowering after pathogen infection. Based on this lack of evidence for increased levels of virulence one could conclude that selection favours low virulent pathogens in clonal plants. For pathogens this would imply that the benefits of potential long-term infections outweigh the costs of possible multiple infections. However, the lack of evidence for clonal growth promoting the evolution of increasingly virulent pathogens can only suggest, but does not necessarily prove that clonality selects for decreased levels of virulence. To our knowledge, no comparative studies of virulence levels between shortlived and long-lived plants, infected with the same pathogen, have been conducted so far. Neither did we find any studies that analyze in detail the effect of pathogen infection on the timing and extent of sexual reproduction in clonal plants. Future research on these topics, empirical studies on multiple infections, and modelling work applying virulence evolution theories for long-lived organisms are hence called for. In this context clonal plants could be essential since they provide ideal systems to test these hypotheses.

Ultimately, the question remains what virulence levels can be expected in pathogens of clonal plants. Not only are the pathogens themselves subjected to conflicting selections pressures, their host in return may have to cope with a range of pathogen virulence levels as well. This interaction between pathogen and host combined with the contrasting selection pressures on virulence mentioned above, will make it even more difficult to define one comprehensive prediction on expected pathogen virulence in clonal plants. Difficult, but all the more intriguing.

Acknowledgements The authors thank Hans de Kroon and two anonymous referees for their comments on a previous version of this manuscript.

\section{References}

Andre JB, Hochberg ME (2005) Virulence evolution in emerging infectious diseases. Evolution 59(7): 1406-1412

Boots M, Mealor M (2007) Local interactions select for lower pathogen infectivity. Science 315:1284-1286

Brown NF, Wickham ME, Coombes BK, Finlay BB (2006) Crossing the line: Selection and evolution of virulence traits. Plos Pathol 2(5):346-353 
Brown SP, Hochberg ME, Grenfell BT (2002) Does multiple infection select for raised virulence? Trends Microbiol 10(9):401-405

Cook RE (1985) Growth and development in clonal plant populations. In: Jackson JBC (ed) Population biology and evolution of clonal organisms. Yale University Press, New Haven, pp 259-296

Day T (2003) Virulence evolution and the timing of disease life- history events. Trends Ecol Evol 18(3):113-118

Day T, Proulx SR (2004) A general theory for the evolutionary dynamics of virulence. Am Nat 163(4):E40E63

Eriksson O (1993) Dynamics of genets in clonal plants. Trends Ecol Evol 8(9):313-316

Ewald PW (1994) Evolution of infectious disease. Oxford University Press, New York

Frank SA (1996) Models of parasite virulence. Q Rev Biol 71(1):37-78

Gandon S, Jansen VAA, van Baalen M (2001) Host life history and the evolution of parasite virulence. Evolution 55(5):1056-1062

Hamilton WD, Axelrod R, Tanese R (1990) Sexual reproduction as an adaptation to resist parasites (A Review). Proc Natl Acad Sci USA 87(9):3566-3573

Kemperman JA, Barnes BV (1976) Clone size in American aspens. Can J Bot 54:2603-2607

Lipsitch M, Moxon ER (1997) Virulence and transmissibility of pathogens: what is the relationship? Trends Microbiol 5(1):31-37

May RM, Nowak MA (1995) Coinfection and the evolution of parasite virulence. Proc R Soc Lond B Biol Sci 261(1361):209-215

Nowak MA, May RM (1994) Superinfection and the Evolution of Parasite Virulence. Proc R Soc Lond B Biol Sci 255(1342):81-89

Oinonen E (1967) The correlation between the size of Finish bracken (Pteridium aquiliunum (L.) Kuhn.) clones and certain periods of history. Acto Forestal Fenn 83:1-51

Steinger T, Körner C, Schmid B (1996) Long-term persistence in a changing climate: DNA analysis suggests very old ages of clones of alpine Carex curvula. Oecologia 105:94-99

Stuefer JF, Gómez S, van Mölken T (2004) Clonal integration beyond resource sharing: implications for defence signalling and disease transmission in clonal plant networks. Evol Ecol 18:647-667

Thomas H (2002) Ageing in plants. Mech Ageing Dev 123:747-753

Van Baalen M, Sabelis MW (1995) The dynamics of multiple infection and the evolution of virulence. Am Nat 146(6):881-910 